2019-02

\title{
Molecular changes to the rat renal cotransporters PEPT1 and PEPT2 due to ageing
}

\section{Alghamdi, OA}

http://hdl.handle.net/10026.1/11976

\subsection{7/s11010-018-3413-x \\ Molecular and Cellular Biochemistry \\ Springer Verlag}

All content in PEARL is protected by copyright law. Author manuscripts are made available in accordance with publisher policies. Please cite only the published version using the details provided on the item record or document. In the absence of an open licence (e.g. Creative Commons), permissions for further reuse of content should be sought from the publisher or author. 


\section{Molecular changes to the rat renal cotransporters PEPT1 and PEPT2 due to ageing}

Othman A. Alghamdi, ${ }^{1,4}$ Nicola King, ${ }^{2}$ Nicholas M. Andronicos, ${ }^{1}$ Graham L. Jones, ${ }^{1}$ Belal Chami, ${ }^{3}$

Paul K. Witting, ${ }^{3}$ Pierre D.J. Moens ${ }^{1}$

${ }^{1}$ School of Science and Technology, University of New England Armidale, NSW 2351, Australia

${ }^{2}$ School of Biomedical and Healthcare Sciences, University of Plymouth, Plymouth PL4 8AA, UK

${ }^{3}$ Discipline of Pathology, Sydney Medical School, Charles Perkins Centre, The University of Sydney, NSW 2006, Australia

${ }^{4}$ Faculty of Science, Department of Biological Sciences, Centre for Science and Medical Research, University of Jeddah, KSA

Correspondence to: Nicola King

School of Biomedical and Healthcare Sciences, University of Plymouth, Plymouth, PL4 8AA, UK

Email: Nicola.king@plymouth.ac.uk

Tel.: +441752584969

Orcid: 0000-0001-6989-5760

\section{Acknowledgments}

We would like to thank Mr. Brian Cross and Mr. Jonathon Clay for their excellent technical assistance. This work was supported by University of New England (unnumbered grant), Australian Research Council (DP160102063) and University of Jeddah (unnumbered grant). 


\begin{abstract}
Renal PEPT1 and PEPT2 cotransporters play an important role in the balance of circulating body oligopeptides and selected peptidomimetic drugs. We aim to comprehensively characterise age-related changes of the renal PEPT cotransporters at the gene, protein, and functional level. Brush border membrane vesicles (BBMV) and outer medulla membrane vesicles (OMMV) were isolated from the kidneys of young, middle-aged and old rats. The protein expression of PEPT1 was not only increased in BBMV from old rats, but PEPT1 also appeared in OMMV from middle-aged and old rats. SLC15A1 gene expression in the renal cortex increased in middle-aged group. PEPT2 protein expression was not only increased with ageing, but PEPT2 also was found in BBMV from middle-aged and old groups. SLC15A2 gene expression in the renal outer medulla increased in the old group. These changes in the expressions and localisations of PEPT1 and PEPT2 could explain the changes to transport activity in BBMV and OMMV. These findings provide novel insights that would be useful for maintaining protein nutrition and optimising the delivery of some peptidomimetic drugs in elderly individuals.
\end{abstract}

Key Words: Ageing / Oligopeptide transport / PEPT1 / PEPT2 / kidney. 


\section{Introduction}

The percentage of people over 65 years of age was about $11 \%$ of the world population in 2007. This percentage was predicted to progressively increase to $22 \%$, of which one individual in every five would be 80 years old in 2050 [1]. Ageing is not a single condition but rather a representative term for the cumulative negative changes at the molecular, cellular and tissue level [2]. Multiple organs and systems, mainly the: kidney, liver, intestine, cardiovascular, and central nervous system undergo progressive functional impairments with ageing [3]. The effect of ageing on renal function and morphology has been extensively studied in different ways and in a wide range of subjects. Most of these studies reported major changes in kidney structure and concomitant decline in renal function [4-5]. For example, after the age of 40 , nephron mass and renal blood flow start to decrease by $10 \%$ per decade and whilst the normal glomerular filtration rate (GFR) is $120 \mathrm{~mL} / \mathrm{min}$, it can decline to $\sim 60-70 \mathrm{~mL} / \mathrm{min}$ at age 85 [6]. However, the age-related decrease in GFR is still being debated, because of the coexistence of chronic kidney disease (CKD) in some elderly people [5]. Comorbidity of ageing and other diseases (e.g. diabetes and hypertension) occurs in most elderly individuals, which can affect the quality of life and can increase the risk of mortality [1].

The initial portion of the nephron, namely the renal proximal tubule is the main site of reabsorption of almost all of the filtered organic compounds including amino acids. The percentage of small molecular weight peptides present in the circulating plasma is not clear yet, but it has been reported that approximately $50 \%$ of all amino acids circulate in the form of di-and-tripeptides [7]. The reabsorption of any filtered or enzymatically hydrolysed di-andtripeptides by PEPT1 and PEPT2 cotransporters in the proximal tubule of the kidney is a pivotal process in balancing protein intake according to the body needs. In addition to the 
capability of PEPT1 and PEPT2 to transport approximately 8000 potential combinations of di-and- tripeptides, they are also able to translocate peptidomimetic drugs with clinical and pharmacological relevance. These include $\beta$-lactam antibiotics of the aminocephalosporin (e.g., Cefadroxil) and Penicillin (e.g., Ampicillin) classes; some antiviral (e.g., Valacyclovir); and anti-tumour drugs (e.g., $\delta$-amino-levulinic acid and $\gamma$-glutamyl-L-cysteine); and selected angiotensin converting enzyme (ACE) inhibitors (e.g., captopril) [8-9].

Age-related changes in physiology can lead to compromised pharmacokinetics and pharmacodynamics including a reduction in renal and hepatic clearance and an increase in the volume of distribution of some drugs (e.g., ACE inhibitors). This can result in prolongation of drug half-life, which could be toxic to the system (reviewed in [1-2]). It has been emphasised that drug dosage must be appropriately adjusted in individuals with renal dysfunction to avoid further complication and adverse reactions $[4,10]$. Therefore, the agedependent decline in renal function could have significant implications, not only on physiological homeostasis, but also on therapy and drug handling [4].

Given that PEPT1 and PEPT2 cotransporters have been implicated in the transport of a wide range of di- and tripeptides besides many important peptidomimetic drugs, our hypothesis is that ageing affect these transporters at the molecular and functional level. This study provides the first experimental evidence for the effect of ageing on PEPT1 and PEPT2 cotransporters in the kidney by comprehensively investigating the changes of localisation, gene and protein expression as well as corresponding protein function. 


\section{Material and Methods}

\section{Ethical Approval}

This study was approved by the Animal Ethics committee of the University of New England, and complies with the Guide for the care and use of laboratory Animals published by the US National Institute of Health (NIH Publication No. 85-23, revised 1996).

\section{Chemicals}

All chemicals used in this study were of analytical quality. $\beta$-Ala-Lys (AMCA) was from Bio Trend Chemicals (Destin, USA). The antibodies: PEPT1, PEPT2, and $\beta$-Actin were from SANTA CRUZ. Primers were purchased from GeneWorks and Sigma. TSA amplification kit was from PerkinElmer. Sudan Black B was from Sigma.

\section{Kidneys of Animal Models}

The age categories of the male Wistar rat groups were chosen according to the criteria described previously by Sengupta [11]: young adult group (6-7 months), middle-aged group (17-18 months) and old group (23-24 months). Kidneys were removed from these groups after stunning and cervical dislocation. The kidneys were snap frozen in liquid nitrogen prior to transfer to $-80{ }^{\circ} \mathrm{C}$ until used for the experiments. Some of these snap-frozen kidneys were transferred to the Charles Perkins Centre at the University of Sydney for conducting part of the immunohistofluorescence studies. 


\section{Isolation of Brush Border Membrane (BBMV) and Outer Medulla Membrane (OMMV) Vesicles and their quality assessment}

BBMV were prepared from 1-2 mm thick slices of superficial cortex from the kidneys of Wistar rat groups, while OMMV were sliced from pieces of the outer medulla, which are characterised by red stripes on visual inspection of the tissue samples [12]. The BBMV and OMMV were prepared by dual-stage $\mathrm{Mg}^{++}$precipitation in the presence of EGTA as described previously [13]. After isolation, vesicles were suspended with intra-vesicular medium containing in mM: 100 mannitol, $100 \mathrm{KH}_{2} \mathrm{PO}_{4}$ and 10 HEPES (pH 7.4 with TRIS) and centrifuged at $31,000 \times \mathrm{g}$ for $20 \mathrm{~min}$ at $4{ }^{\circ} \mathrm{C}$. The resulting pellets were re-suspended in the same buffer $(500 \mu \mathrm{L})$ and pre-equilibrated using the freeze-thaw fractionation method [14]. The quality of the isolated BBMV and OMMV was verified by measuring the enzyme activities and enrichments of leucine aminopeptidase (LA) and alkaline phosphatase (AP) [13]. The enrichments of LA and AP in all preparations were higher than 10-fold (see table 1 in supplementary file). These values are comparable with previous studies and indicate that the procedure yielded high-quality and uncontaminated vesicles [13, 15]. The protein concentration of each vesicular isolate was quantified using Bradford's method as described previously [16].

Several measures were undertaken to minimise adventitial oxidation in these preaprations. While dissecting the kidneys, renal slices were transferred to ice-cold buffer containing (in mM): 300 Mannitol, 5 Ethylen glycol tetraacetic acid (EGTA), and 10 2-[4-(2hydroxymethyl)-aminomethan (TRIS)). Both EGTA and mannitol are antioxidants; EGTA binds various divalent cations (including $\mathrm{Fe}$ and $\mathrm{Cu}$ ) to render these metals redox inactive [17], and mannitol can scavenge potent one-electron oxidants such as hydroxyl radical [18]. BBMV and OMMV isolated by the method of Biber et al. [13] illustrated are devoid of contamination by mitochondria and endoplasmic reticulumindicating that these vesicles lack 
intracellular machinery components that promote ROS generation. Finally, after isolation all vesicles were immediately snap frozen in liquid nitrogen prior to storage at $-80^{\circ} \mathrm{C}$ until use (within 4 weeks of isolation).

\section{Fluorescence measurements in BBMV and OMMV}

The transport activity of PEPT1 and PEPT2 in BBMV and OMMV were measured using the fluorophore-conjugated dipeptide $\beta$-Ala-Lys (AMCA) as we described previously [19]. Briefly, $\beta$-Ala-Lys (AMCA) was prepared to its maximum soluble concentration $(2.32 \mathrm{mM}$ according to BioTrend) in the extra-vesicular buffer containing in mM: $100 \mathrm{KH}_{2} \mathrm{PO}_{4}, 100$ mannitol, and 10 2-(N-Morpholino) ethanesulfonic acid (MES)tris(hydroxymethyl)aminomethane (TRIS) ( $\mathrm{pH}$ 6.6). Vesicles containing $45 \mu \mathrm{g}$ of total proteins $(\sim 15 \mu \mathrm{L})$ were added to 7 different concentrations of $\beta$-Ala-Lys-AMCA (ranging from 5 to $2000 \mu \mathrm{M}$ ) with extra-vesicular buffer added to yield a final reaction volume of 250 $\mu \mathrm{L}$ into Eppendorf tubes. After incubation for $20 \mathrm{~min}$ at $20^{\circ} \mathrm{C}$, the transport of the substrate was stopped by transferring the mixture into centrifuge tubes containing $5 \mathrm{~mL}$ of ice-cold extra-vesicular buffer prior to spinning at $31,000 \times \mathrm{g}$ for $20 \mathrm{~min}$ at $4{ }^{\circ} \mathrm{C}$. The resulting pellets were washed 3 times with the extra-vesicular buffer $(3 \mathrm{~mL})$ and then resuspended in $500 \mu \mathrm{L}$ of the same buffer. Reaction mixtures were then transferred into 96-well black microplates using $166 \mu \mathrm{L}$ in each well arranged in triplicates. Fluorescence emission was measured at 455 $\mathrm{nm}$ at an excitation wavelength of $350 \mathrm{~nm}$ using a microplate reader (SpectraMax-M2 ${ }^{\mathrm{e}}$ ). Each plate contained vesicles isolated from the three designated age groups so that all measurements were matched and taken under the same experimental conditions. 


\section{Immunofluorescence study}

Snap-frozen kidney samples embedded in Optimal Cutting Temperature Compound (OCT) were cut into 7 micron sections using a cryostat (Leica CM 1850) maintained at $-18{ }^{\circ} \mathrm{C}$. Three sections from three different groups (young, middle-aged, and old) were mounted onto Superfrost Plus slide (Thermo Scientific) and air-dried for $30 \mathrm{~min}$ at $22{ }^{\circ} \mathrm{C}$. A control was prepared for each specimen on a separate Superfrost Plus slide. The slides were then wrapped with aluminium foil prior to transferring to a $-80{ }^{\circ} \mathrm{C}$ freezer for later use. In this way, the tissue architecture was partially protected from moisture damage when taking them out again for staining. Kidney tissues are known to produce high autofluorescence (AF), which can be an obstacle in detecting specific antibody-labelled immunofluorescence probe. Sun et al. [20] showed that $0.1 \%(\mathrm{w} / \mathrm{v})$ of Sudan Black B (SBB) was effective in blocking intense AF arising from the kidney tissues of normal and nephropathy mice without adversely interfering with the specific immunofluorescence signal in frozen sections. In this study, it was found that AF was high especially in the kidney tissues of middle-aged and old rats; hence it was required to optimise for the best percentage of SBB (ranging from 0.1 to $0.5 \% \mathrm{w} / \mathrm{v}$ ), of which $0.3 \% \mathrm{w} / \mathrm{v}$ was ascertained to be effective in blocking AF (see Figure 1 in supplementary file). In addition, Tyramide Signal Amplification (TSA) Systems (PerkinElmer), which is an enzyme-mediated detection utilising HRP to generate high-density labelling with sensitivity up to 100 -fold higher than conventional method, was used in this experiment as the affinity of the fluorescently labelled antibodies was affected. By this method, it was also possible to probe the tissues with the same antibodies used in Western blotting.

Where required, the foil-wrapped slides were taken out of the freezer and equilibrated at $22{ }^{\circ} \mathrm{C}$ for $30 \mathrm{~min}$ prior to fixing in ice-cold acetone for $20 \mathrm{~min}$. After that, they were gently washed with water and incubated with $0.3 \% \mathrm{v} / \mathrm{v} \mathrm{H}_{2} \mathrm{O}_{2}$ for 15 min to quench endogenous 
peroxidase activity. The slides were washed 3 times with TBS-T buffer containing in $\mathrm{mM}$ : $150 \mathrm{NaCl}, 20$ Tris- $\mathrm{HCl}(\mathrm{pH} 7.5)$ and $0.1 \%$ Tween 20, followed by $1 \mathrm{~h}$ incubation with either 1:400 (v/v) mouse monoclonal anti-PEPT1 or goat polyclonal anti-PEPT2 for $1 \mathrm{~h}$ at RT. This was followed by 3 times washing with TBS-T and incubation with secondary antibodies goat/ antimouse or mouse/ antigoat accordingly in 1:300 (v/v) dilution. After removing the secondary antibodies by washing 3 times with TBS-T, 1:50 (v/v) TSA-CY 3.5 was added to the slides for $10 \mathrm{~min}$, followed by washing 3 times with TBS-T prior to applying SBB $(0.3 \%$ $\mathrm{w} / \mathrm{v}$ in $70 \% \mathrm{v} / \mathrm{v}$ ethanol/ $\mathrm{H}_{2} \mathrm{O}$ ) for $30 \mathrm{~min}$. Finally, the sections were washed with TBS-T for 10 min and then mounted with Dako cytomation fluorescent mounting medium containing DAPI (Dako, USA) and placed under a coverslip. Control slides containing the same sections were used to confirm the suppression of AF in SBB-treated sections, and to examine the specificity of the staining by probing the sections with only secondary antibodies (no primary antibody). A TI eclipse (Nikon corporation, Tokyo, Japan) equipped with a colour cooled DXM1200C digital camera was used for fluorescence imaging. The optimum conditions of gain and exposure time were established in a preliminary experiment based on the AF level of untreated and SBB-treated sections of all age groups. Exposure time was set to $300 \mathrm{~ms}$ and the gain was set to 6.3 . Images of resolution $1280 \times 1024$ px were acquired for the blue, green, red channels and a brightfield.

\section{Western blotting}

BBMV and OMMV isolated from the kidneys of each age group were used to probe for PEPT1 and PEPT2 proteins using Western blotting as described previously [21]. Briefly, 35 or $45 \mu \mathrm{g}$ proteins of these vesicle preparations were separated on a SDS-PAGE Mini-Protean Gels (4-20\%, Bio-Rad) and transferred on a nitrocellulose membrane. After blocking the 
membranes overnight with $5 \% \mathrm{w} / \mathrm{v}$ skim milk dissolved in TBS-T buffer, they were probed with either mouse monoclonal anti-PEPT1 or goat polyclonal anti-PEPT2. This was followed by probing with Horse radish peroxidase-conjugated goat anti-mouse and mouse anti-goat. Following the probing stages by primary and secondary antibodies and after incubation with the secondary antibodies, the membranes were washed three times for 10 min with TBS-T buffer. After that, ECL ${ }^{\mathrm{TM}}$ Western Blotting Analysis System (GE Healthcare) was used according to the manufacturer's instructions to image the protein bands on Hyperfilm ${ }^{\mathrm{TM}} \mathrm{ECL}$ films (GE Healthcare). The same membranes were then stripped and re-probed with mouse monoclonal anti- $\beta$-Actin. The intensity of protein bands were quantified using BIO-RAD GEL DOC EQ system and its associated software (Quantity One. inc).

\section{RT-PCR and real-time RT-qPCR}

Biopsies of renal tissues $(18 \mathrm{mg}$ ) were removed from the superficial cortex and outer medulla of renal tissue for each age group (samples were taken from kidneys previously snap-frozen for storage). Total RNA was isolated from these tissues using ISOLATE II RNA Mini Kit (BIOLINE, Cat No: Bio-52072). Three quality control experiments were performed prior to proceeding to cDNA synthesis: (1) the RNA purity and quantity was checked by measuring the absorbance at 260/280 ratio >1.9 with a NanoDrop UV-Vis Spectrophotometer (Thermo Scientific); (2) the RNA integrity was confirmed by $1 \%$ w/v agarose gel where the intensity of tRNA $28 \mathrm{~S}$ was twice that of the $18 \mathrm{~S}$ bands; (3) possible contamination by genomic DNA was checked by adding $10 \mathrm{ng}$ of RNA from each sample to $0.5 \mu \mathrm{M}$ forward and reverse PEPT primers, $5 \mu \mathrm{L}$ SYBR green, and $3 \mu \mathrm{L}$ nuclease-free water. These experiments, which were run under the same conditions as described below for the cDNA samples did not show any amplification, indicating good RNA isolation (see Figure 2 in supplementary file). 
cDNA synthesis of isolated total RNA samples was performed in 96-well plates ( $\mathrm{T}$ $100^{\mathrm{TM}}$ Thermal Cycler, Bio-Rad) using Tetro cDNA Synthesis Kit (BIOLINE). Total RNA (5 $\mu \mathrm{g}$ ) was mixed with Oligo (dT) 18 , Random Hexamer, dNTP mix, RiboSafe RNase Inhibitor, and Tetro Reverse Transcriptase according to the manufacture instructions. The resulting cDNA was then used to perform q-PCR, by mixing $5 \mu \mathrm{L}_{\text {SensiFAST }}^{\mathrm{TM}}$ SYBR (Bioline) with cDNA (10 ng RNA; the use of RNA concentration as the concentration for cDNA is recommended and since the isolated RNA was diluted in RNAse free water, it was possible to blank against this water using a nanodrop spectrophotometer) and $500 \mathrm{nM}$ forward and reverse primers (Table 1) in a 384-well plate (in duplicate). Primers for $\beta$-Actin, and SDHA (succinate dehydrogenase) were found to be the most stable internal reference genes in rat kidneys with ageing, but not TBP (TATA Box binding Protein) nor GAPDH (glyceraldehyde 3-phosphate dehydrogenase) in this experiment. The loaded 384-well plate was read on a QRT-PCR Bio-Rad CFX plate reader. This was started by denaturation at $95{ }^{\circ} \mathrm{C}$ for $2 \mathrm{~min}$, followed by amplification for 39 cycles of denaturation, annealing and extension as follows: $95^{\circ} \mathrm{C} / 10 \mathrm{~s}, 60^{\circ} \mathrm{C} / 20 \mathrm{~s}, 72^{\circ} \mathrm{C} / 15 \mathrm{~s}$. These reactions were always followed by 1 cycle step to generate the melt curve at $95^{\circ} \mathrm{C} / 0.5 \mathrm{~s}, 65^{\circ} \mathrm{C} / 0.5 \mathrm{~s}, 95^{\circ} \mathrm{C} / 5 \mathrm{~s}$. The $\Delta \Delta \mathrm{Cq}$ method of the Bio-Rad CFX Manager 3.1 was used to determine differences between samples by normalising with two reference genes. All samples produced single peaks, indicating specific product amplification. The predicted sizes of products were examined by running samples $(5 \mu \mathrm{L})$ on $2 \%$ agarose gel, which showed the expected sizes. Before these steps, two samples were only converted to cDNA to check the specificity of the primers. The MIQE guidelines for the minimum information of RT-qPCR experiments were followed in this study [22].

\section{Statistical Analysis}


Data from transport experiments and relative measurements of protein and gene level were expressed as means \pm SE of $\mathrm{n}$ experiments. Students $\mathrm{T}$ test or one-way ANOVA as appropriate were used to test for significant differences. A $p$ value $<0.05$ was considered significant.

\section{Results}

\section{Kinetic measurements of $\beta$-Ala-Lys (AMCA) uptake}

The fluorescence intensity changes following incubation with $\beta$-Ala-Lys (AMCA) for the BBMV and OMMV obtained from kidneys isolated from the different age groups (young adult, middle-aged and old rats) were plotted versus increasing $\beta$-Ala-Lys (AMCA) concentration. The points were then curve fitted using the Michaelis-Menten equation. The Michaelis-Menten curves for $\beta$-Ala-Lys (AMCA) uptake into BBMV and OMMV isolated from the kidneys of all rat groups are shown with corresponding curve-fits (Figure 1 and 2; $\mathrm{R}^{2}>0.9$ ). An effect of ageing on $\beta$-Ala-Lys (AMCA) transport by BBMV and OMMV was clearly seen when comparing the transport kinetic parameters $K_{m}$ and $V_{\max }$ (Table 2). In the young group, OMMV showed significantly higher affinity than BBMV ( $\mathrm{P}=0.0004)$, but significantly lower capacity than BBMV $(\mathrm{P}=0.0003)$. By contrast, middle-aged and old groups did not show any significant differences in the affinity or capacity between BBMV and OMMV. The affinity of $\beta$-Ala-Lys (AMCA) uptake by BBMV in middle-aged and old groups was significantly higher than BBMV in young adult group (both $\mathrm{P}=0.001$ ), however the capacity was significantly lower than BBMV in young adult group (both $\mathrm{P}=0.0001$ ). In addition, the affinity of OMMV to $\beta$-Ala-Lys (AMCA) in middle-aged was significantly higher than OMMV in young adult group $(\mathrm{P}=0.01)$, but the capacity was significantly lower 
than OMMV in young adult group $(\mathrm{P}=0.005)$. No significant differences in affinity or in capacity was determined between middle-aged and old groups (Table 2).

\section{Localisation of PEPT1 and PEPT2 in the rat kidney with ageing}

The normal distribution of PEPT1 and PEPT2 cotransporters along the proximal tubule was reported previously to be exclusively situated in Segments 1 and 3 respectively [12, 23]. As shown in Figure $3(\mathrm{~A}, \mathrm{G})$, PEPT1 is present in the superficial cortex region of young kidneys, but not in deeper segments as opposed to the middle-aged and old rats which revealed a progressively wider spread of PEPT1 distribution (3B-C, H-I) in all segments of the proximal tubule and medulla. Similarly, red-PEPT2 specific staining was most intense in the outer medulla of the young group with progressively weaker staining in the cortex and medulla $(4 \mathrm{~A}, \mathrm{G})$, as opposed to middle-aged and old groups that showed a more consistent positive staining intensity in the cortex and medulla (Figure 4 B-C, H-I). Notably, both transporters seem to be present in the kidney glomeruli of middle-aged and old groups as judged by immune-positivity in these kidney regions.

\section{Protein expression of PEPT1 and PEPT2 in kidney membrane vesicles}

Western blot assay was used in this experiment to relatively quantify the presence of PEPT cotransporters in two main regions of the renal proximal tubule of different age groups of Wistar rats. As shown in Figures 5 and 6, both BBMV and OMMV were probed with antiPEPT1 and anti-PEPT2 antibodies. The bands intensities of these proteins together with their corresponding $\beta$-actin bands were quantified and the results were summarised in Figure 7. 
These data indicated that there were significant differences in PEPT1 and PEPT2 protein levels in all age groups (ANOVA, $\mathrm{P}=0.03$ and $7 \times 10^{-8}$ respectively). The presence of PEPT1 in OMMV and PEPT2 in BBMV (i.e., regions where these proteins are reported to be absent) from old and middle-aged groups was also confirmed in these experiments and verifies the immuno-histochemical data above. This was followed by post-hoc t-test which indicated a significant increase in the intensity ratio of PEPT1 protein in BBMV of old group (Figure 7A): PEPT1 in young BBMV vs. PEPT1 in old BBMV (P=0.04); PEPT1 in middleaged BBMV vs. PEPT1 in old BBMV ( $\mathrm{P}=0.01)$; $\mathrm{PEPT} 1$ in middle-aged BBMV vs. PEPT1 in middle-aged and old OMMV ( $\mathrm{P}=0.03)$. However, no significant differences were seen between young BBMV vs. middle-aged BBMV and OMMV. Further, the intensity ratios of PEPT2 protein in OMMV and BBMV showed significant changes (Figure 7B): PEPT2 in young OMMV vs. PEPT2 in middle-aged OMMV and BBMV $\left(\mathrm{P}=0.001\right.$, and $\left.4 \times 10^{-5}\right)$, and vs. PEPT2 in old OMMV and BBMV $\left(\mathrm{P}=2 \times 10^{-5}\right.$ and $5 \times 10^{-5}$ respectively). Also, PEPT2 in middle-aged OMMV vs. PEPT2 in middle-aged BBMV $(\mathrm{P}=0.001)$ and vs. PEPT2 in old OMMV and BBMV $(\mathrm{P}=0.005$, and 0.001 respectively).

\section{RNA expression of PEPT1 and PEPT2 with ageing}

The expression of SLC15A1 and SLC15A2 genes encoding PEPT1 and PEPT2 transporters respectively, in the superficial cortex and outer medulla of the renal proximal tubules were then determined with quantitative PCR. The relative normalised values extracted from BioRad CFX Manager 3.1 were examined by Kolmogorov-Smirnov to test for the non-normality distribution hypothesis. This test showed that these values were normally distributed, hence the analysis approach using ANOVA followed by post-hoc t-test was applied to investigate the differences between groups was validated. As shown in Figure 8, the relative expression 
of SLC15A1 gene in the middle-aged group was significantly upregulated when compared to the young adult group $(\mathrm{P}=0.04)$. However, the old group did not show any significant changes when compared to middle-aged or young adult groups. In addition, the relative expression of SLC15A2 gene in the old age group was significantly higher than both the young adult group and middle-aged group $(\mathrm{P}=0.02$ and 0.04 respectively), but no significant changes were found between the young adult and middle-aged groups. Furthermore, the relative gene expression of SLC15A2 were significantly higher than SLC15A1 in all ages: young adult $(\mathrm{P}=0.04)$, middle-aged $(\mathrm{P}=0.005)$, and old group $(\mathrm{P}=0.0007)$.

\section{Discussion}

The major findings of this investigation were that the $\mathrm{K}_{\mathrm{m}}$ and $\mathrm{V}_{\max }$ of $\beta$-Ala-Lys (AMCA) transport into BBMV and OMMV isolated from rat kidneys differed between young, middleaged and old rats (Fig. 1-2 and table 2). These changes could possibly be explained by a redistribution of the PEPT transporters in the kidney with ageing. This was demonstrated by the appearance of PEPT1 protein in the outer medulla and medulla of middle-aged and old rat kidneys (Fig. 3, 6-7), whilst PEPT2 protein was expressed in the cortex of middle-aged and old rat kidneys (Fig. 4, 6-7). In addition, the SLC15A2 (PEPT2) gene showed a significant increase in the old group compared to young and middle-aged groups (Fig. 8).

One of the main results of this study was the redistribution of the renal PEPT transporters with ageing (Fig. 3-4, 6-7). The underlying mechanism could involve a compensatory process, which balances the loss of nephron mass and decreased glomerular filtration rate that is known to occur with ageing [5, 24]. Supporting evidence that PEPT transporters can be re-distributed in the kidney was obtained by Tramonti et al [25]. They 
showed that following uninephrectomy PEPT1 and PEPT2 were expressed in all segments of the proximal tubule.

The kinetic experiments described in our manuscript represent the uptake of $\beta$-AlaLys (AMCA) into BBMV and OMMV isolated from the kidneys of different aged rats. In BBMV from young rats there is only a single transporter present, PEPT1. In OMMV from young rats there is only PEPT2. However in the BBMV and OMMV isolated from the kidneys of middle-aged and old rats both PEPT1 and PEPT2 are present. This explains the changes in capacity and affinity of $\beta$-Ala-Lys (AMCA) uptake in the two vesicle groups (table 2).

The relationship between the intestinal PEPT transporters and ageing has been subject to limited investigation before. Knock-out of the pep2 (equivalent of PEPT1) gene in Caenorhabditis elegans did not have any effect on the lifespan [26]. Also with regard to intestinal PEPT transporters it has been shown that $\beta$-Klotho causes down-regulation, which it was speculated could have an impact on ageing [27]. It should be noted, however, that unlike the current study neither of these studies directly investigated the effect of ageing on PEPT transporter expression and function in the kidney.

Considering the nutritional and clinical implications of PEPT1 and PEPT2 transporters, these results suggest that ageing may affect the renal handling of the hydrolysed proteins and peptide-like drugs including $\beta$-lactam antibiotics and some ACE inhibitors (used as anti-hypertensive agents). This could for example, make the management of hypertension in elderly patients more complicated than in the young as dose adjustment of hypertensive agents maybe required to avoid adverse effects [28-30].

Age-related changes in renal structure and function are often accompanied by one or more pathological conditions (e.g. hypertension, diabetes), which could lead to the instability 
of individual homeostasis affecting renal handling of nutrients and drugs [4]. The 5/6 nephrectomised rat has been widely used as an experimental model for the progression of renal damage and chronic renal failure which resemble the effect of ageing on the kidney based on the following: (i) reduction of nephron mass, (ii) decreased glomerular filtration, (iii) presence of proteinuria, (iv) functional hypertrophy, and (v) morphological changes to the glomerulus [5, 24]. A study conducted by Takahashi et al. [31] showed that the protein and gene expression of PEPT2 significantly increased in the $5 / 6$ nephrectomised rats, resulting in a 3-fold increase in $\mathrm{V}_{\max }$, but without any changes in $\mathrm{K}_{\mathrm{m}}$ values as determined by functional activity studies. These findings are in agreement with this study of the age-related changes of PEPT cotransporters at the gene and protein expression levels.

In addition to compensating for nephron loss and decreased glomerular filtration rate the change in PEPT transporters with ageing could also compensate for a changing hormonal and oxidant environment. It has been shown that leptin [32-33], growth hormone [35] and insulin $[29,36]$ stimulate the expression and activity of PEPT1. While the transport activity and expression of PEPT1 significantly decreased in Caco-2 cells challenged by the oxidant $\mathrm{H}_{2} \mathrm{O}_{2}$ [35], Caco-2 cells pre-treated with the thyroid hormone 3,5,3'-L-triiodothyronine ( $\left.\mathrm{T}_{3}\right)$ [37], and hyperthyroid rats [38] demonstrated significantly higher gene and protein expression of the renal PEPT2 compared to the control rats [39]. With ageing comes leptin resistance [40] and increased insulin resistance coupled with glucose intolerance (reviewed in [41]), and decreased level of thyroid stimulating hormone (TSH) and T3 in the serum of elderly individuals (reviewed in [42]), which could play a role in the regulation of PEPT1 and PEPT2 cotransporters.

Notably, ageing is not a single condition, but rather a term representative of cumulative changes at molecular, cellular, and tissue level [2]. The concept of progressive damage caused by age-dependent increases in ROS generation is one of the established 
theories to explain why ageing occurs [6]. Thus, unregulated generation of ROS is associated with the pathogenesis of different chronic diseases such as atherosclerosis, hypertension, adiposity, cancer, and type II diabetes [43]. The age-related alterations on the PEPT1 and PEPT2 cotransporters identified here have the potential to impact renal metabolism of ditripeptides. Some amino acids, both in the form of single amino acids [44-45] or ditripeptides [46-48] play an important role as ROS scavengers such as singlet oxygen and ${ }^{\circ} \mathrm{OH}$ radicals, lipid peroxyl radicals, that further exacerbate ROS-mediated damage in ageing tissues. The scope of this present study was to investigate the effect of ageing on these cotransporters at the molecular level and we demonstrate that ageing has significant bearing on the expression level and renal distribution of these cotransporters. Confirming that other pathologies also impacted by altered renal cotransporter levels and tissue distribution is a relevant question that warrants further work in order to define their implications for PEPT cotransporters in different disease states.

In conclusion, these findings and discussions may provide useful insights for the maintenance of nutritional amino acids and dosage optimisation of peptide-like drugs in the elderly and could help understanding of the underlying mechanism(s) regulating PEPT cotransporters and the relative change in co-transporter function in subjects from different age groups.

\section{Conflict of interest}

The authors declare that they have no conflict of interest.

\section{Ethical approval}


All applicable international, national, and/or institutional guidelines for the care and use of animals were followed. 


\section{References}

[1]. Shi S, Morike K \& Klotz U. (2008). The clinical implications of ageing for rational drug therapy. European Journal of Clinical Pharmacology, 64, 183-199.

[2]. Mangoni AA \& Jackson SH. (2004). Age-related changes in pharmacokinetics and pharmacodynamics: basic principles and practical applications. British Journal of Clinical Pharmacology, 57, 6-14.

[3]. Aalami OO, Fang TD, Song HM \& Nacamuli RP. (2003). Physiological features of aging persons. Archives of Surgery 138, 1068-1076.

[4]. Martin JE \& Sheaff MT. (2007). Renal ageing. The Journal of Pathology, 211, 198-205.

[5]. Weinstein JR \& Anderson S. (2010). THE AGING KIDNEY: PHYSIOLOGICAL CHANGES. Advances in Chronic Kidney Disease 17, 302-307.

[6]. Arking R. (2006). The Biology of Aging. Oxford University Press New York.

[7]. Seal CJ \& Parker DS. (1991). Isolation and characterization of circulating low molecular weight peptides in steer, sheep and rat portal and peripheral blood. Comparative Biochemistry and Physiology B 99, 679-685.

[8]. Brandsch M, Knutter I \& Bosse-Doenecke E. (2008). Pharmaceutical and pharmacological importance of peptide transporters. The Journal of pharmacy and pharmacology, 60, 543585.

[9]. Anderson CM \& Thwaites DT. (2010). Hijacking solute carriers for proton-coupled drug transport. Physiology (Bethesda, Md) 25, 364-377.

[10]. Schwartz JB. (2007). The current state of knowledge on age, sex, and their interactions on clinical pharmacology. Clinical Pharmacology and Therapeutics, 82, 87-96. 
[11]. Sengupta P. (2013). The Laboratory Rat: Relating Its Age With Human's. International Journal of Preventive Medicine, 4, 624-630.

[12]. Shen H, Smith DE, Yang T, Huang YG, Schnermann JB \& Brosius III FC. (1999). Localization of PEPT1 and PEPT2 proton-coupled oligopeptide transporter mRNA and protein in rat kidney. American Journal of Physiology-Renal Physiology 276, F658-F665.

[13]. Biber J, Stieger B, Haase W \& Murer H. (1981). A high yield preparation for rat kidney brush border membranes. Different behaviour of lysosomal markers. Biochimica et Biophysica Acta 647, 169-176.

[14]. Donowitz M, Emmer E, McCullen J, Reinlib L, Cohen ME, Rood RP, Madara J, Sharp GW, Murer H \& Malmstrom K. (1987). Freeze-thaw and high-voltage discharge allow macromolecule uptake into ileal brush-border vesicles. American Journal of Physiology 252, G723-735.

[15]. Nakano M, Ito Y, Kohtani K, Mizuno T \& Tauchi H. (1985). Age-related change in brush borders of rat kidney cortex. Mechanisms of Ageing and Development 33, 95-102.

[16]. Brown CD, King N \& Simmons NL. (1993). Co-expression of an anion conductance pathway with $\mathrm{Na}(+)$-glucose cotransport in rat renal brush-border membrane vesicles. Pflugers Archiv European journal of physiology 423, 406-410.

[17]. Konopka K. (1978). Differential effects of metal-binding agents on the uptake of iron from transferrin by isolated rat liver mitochondria. FEBS letters 92, 308-312.

[18]. Shen B, Jensen RG \& Bohnert HJ. (1997). Mannitol Protects against Oxidation by Hydroxyl Radicals. Plant Physiology 115, 527-532. 
[19]. Alghamdi OA, King N, Jones GL \& Moens PDJ. (2017). A new use of beta-Ala-Lys (AMCA) as a transport reporter for PEPT1 and PEPT2 in renal brush border membrane vesicles from the outer cortex and outer medulla. Biochim Biophys Acta 1860, 960-964.

[20]. Sun Y, Yu H, Zheng D, Cao Q, Wang Y, Harris D \& Wang Y. (2011). Sudan black B reduces autofluorescence in murine renal tissue. Archives of Pathology \& Laboratory Medicine, 135, 1335-1342.

[21]. Alghamdi OA, King N, Jones GL \& Moens PDJ. (2017). Kinetic Measurements of Diand Tripeptide and Peptidomimetic Drug Transport in Different Kidney Regions Using the Fluorescent Membrane Potential-Sensitive Dye, DiS-C ${ }_{3}-(3)$. J Membr Biol 250, 641-649.

[22]. Bustin SA, Benes V, Garson JA, Hellemans J, Huggett J, Kubista M, Mueller R, Nolan T, Pfaffl MW, Shipley GL, Vandesompele J \& Wittwer CT. (2009). The MIQE guidelines: minimum information for publication of quantitative real-time PCR experiments. Clinical Chemistry, 55, 611-622.

[23]. Smith DE, Pavlova A, Berger UV, Hediger MA, Yang T, Huang YG \& Schnermann JB. (1998). Tubular localization and tissue distribution of peptide transporters in rat kidney. Pharmaceutical Research 15, 1244-1249.

[24]. Hostetter TH, Olson JL, Rennke HG, Venkatachalam MA \& Brenner BM. (1981). Hyperfiltration in remnant nephrons: a potentially adverse response to renal ablation. The American Journal of Physiology, 241, F85-93.

[25]. Tramonti G, Xie P, Wallner El, Danesh FR \& Kanwar YS. (2006). Expression and functional characteristics of tubular transporters: P-glycoprotein, PEPT1, and PEPT2 in renal mass reduction and diabetes. American Journal of Physiology Renal Physiology, 291, F972980. 
[26]. Meissner B, Boll M, Daniel H \& Baumeister R. (2004). Deletion of the intestinal peptide transporter affects insulin and TOR signalling in Caenorhabditis elegans. Journal of Biological Chemistry, 279, 36739-36745.

[27]. Abousaab A, Warsi J, Salker MS \& Lang F. (2016). $\beta$-Klotho as a negative regulator of the peptide transporters PEPT1 and PEPT2. Cell Physiology and Biochemistry, 40, 874-882.

[28]. Amery A, Wasir H, Bulpitt C, Conway J, Fagard R, Lijnen P \& Reybrouck T. (1978). Aging and the cardiovascular system. Acta Cardiologica, 33, 443-467.

[29]. Pinto E. (2007). Blood pressure and ageing. Postgraduate Medical Journal 83, 109114.

[30]. Turnheim K. (2003). When drug therapy gets old: pharmacokinetics and pharmacodynamics in the elderly. Experimental Gerontology, 38, 843-853.

[31]. Takahashi K, Masuda S, Nakamura N, Saito H, Futami T, Doi T \& Inui K. (2001). Upregulation of $\mathrm{H}(+)$-peptide cotransporter PEPT2 in rat remnant kidney. American Journal of Physiology Renal Physiology, 281, F1109-1116.

[32]. Buyse M, Berlioz F, Guilmeau S, Tsocas A, Voisin T, Peranzi G, Merlin D, Laburthe M, Lewin MJ, Roze C \& Bado A. (2001). PepT1-mediated epithelial transport of dipeptides and cephalexin is enhanced by luminal leptin in the small intestine. The Journal of clinical investigation, 108, 1483-1494.

[33]. Hindlet P, Bado A, Farinotti R \& Buyse M. (2007). Long-term effect of leptin on H+coupled peptide cotransporter 1 activity and expression in vivo: evidence in leptin-deficient mice. The Journal of Pharmacology and Experimental Therapeutics, 323, 192-201. 
[34]. Yarandi SS, Hebbar G, Sauer CG, Cole CR \& Ziegler TR. (2011). Diverse roles of leptin in the gastrointestinal tract: modulation of motility, absorption, growth, and inflammation. Nutrition (Burbank, Los Angeles County, Calif) 27, 269-275.

[35]. Alteheld B, Evans ME, Gu LH, Ganapathy V, Leibach FH, Jones DP \& Ziegler TR. (2005). Alanylglutamine dipeptide and growth hormone maintain PepT1-mediated transport in oxidatively stressed Caco-2 cells. The Journal of Nutrition 135, 19-26.

[36]. Thamotharan M, Bawani SZ, Zhou X \& Adibi SA. (1999). Hormonal regulation of oligopeptide transporter pept-1 in a human intestinal cell line. The American Journal of Physiology, 276, C821-826.

[37]. Ashida K, Katsura T, Motohashi H, Saito H \& Inui K. (2002). Thyroid hormone regulates the activity and expression of the peptide transporter PEPT1 in Caco-2 cells. American journal of physiology Gastrointestinal and liver physiology 282, G617-623.

[38]. Ashida K, Katsura T, Saito H \& Inui K. (2004). Decreased activity and expression of intestinal oligopeptide transporter PEPT1 in rats with hyperthyroidism in vivo. Pharmacetical Research 21, 969-975.

[39]. Doring F, Schmitt R, Bernhardt WM, Klapper M, Bachmann S, Daniel H \& Groneberg DA. (2005). Hypothyroidism induces expression of the peptide transporter PEPT2. Biological Chemistry, 386, 785-790.

[40]. Gabriely I, Ma XH, Yang XM, Rossetti L \& Barzilai N. (2002). Leptin resistance during aging is independent of fat mass. Diabetes 51, 1016-1021.

[41]. Chang AM \& Halter JB. (2003). Aging and insulin secretion. American Journal of Physiology-Endocrinology and Metabolism 284, E7-E12.

[42]. Peeters RP. (2008). Thyroid hormones and aging. HORMONES-ATHENS- 7, 28. 
[43]. Aruoma OI, Laughton MJ \& Halliwell B. (1989). Carnosine, homocarnosine and anserine: could they act as antioxidants in vivo? Biochemical Journal 264, 863869.

[44]. King N, McGivan JD, Griffiths EJ, Halestrap AP \& Suleiman MS. (2003). Glutamate loading protects freshly isolated and perfused adult cardiomyocytes against intracellular ROS generation. Journal of Molecular and Cellular Cardiology, 35, 975984.

[45]. Kerksick C \& Willoughby D. (2005). The antioxidant role of glutathione and $\mathrm{N}$-acetyl-cysteine supplements and exercise-induced oxidative stress. Journal of the International Society of Sports Nutrition, 2, 38-44.

[46]. Haynes TE, Li P, Li X, Shimotori K, Sato H, Flynn NE, Wang J, Knabe DA \& Wu G. (2009). L-Glutamine or L-alanyl-L-glutamine prevents oxidant-or endotoxininduced death of neonatal enterocytes. Amino Acids 37, 131-142.

[47]. Tanida M, Shen J, Kubomura D \& Nagai K. (2010). Effects of anserine on the renal sympathetic nerve activity and blood pressure in urethane-anesthetized rats. Physiological Research 59, 177-185.

[48]. Nagai K, Tanida M, Niijima A, Tsuruoka N, Kiso Y, Horii Y, Shen J \& Okumura N. (2012). Role of L-carnosine in the control of blood glucose, blood pressure, thermogenesis, and lipolysis by autonomic nerves in rats: involvement of the circadian clock and histamine. Amino Acids 43, 97-109. 


\section{Figure Legends}

Fig. 1 Fluorescence changes as a function of $\beta$-Ala-Lys (AMCA) concentration following 20 min incubation of BBMV (containing $45 \mu \mathrm{g}$ protein) isolated from the kidneys of young adult (dotted line), middle-aged (solid line), and old (dashed line) rat groups (at $\mathrm{pH}$ 6.6). The curves are fitted to the Michaelis-Menten equation showing $\mathrm{R}^{2}>0.9$. Data shown are means \pm SE, $n=6$ (in triplicate).

Fig. 2 Fluorescence changes as a function of $\beta$-Ala-Lys (AMCA) concentration following 20 min incubation of OMMV (containing $45 \mu \mathrm{g}$ protein) isolated from the kidneys of young adult (dotted line), middle-aged (solid line), and old (dashed line) rat groups (at pH 6.6). The curves are fitted to the Michaelis-Menten equation showing $\mathrm{R}^{2}>0.9$. Data shown are means \pm SE, $n=6$ (in triplicate)

Fig. 3 Microscopy images of kidney cross sections. PEPT1 staining was measured using the immunofluorescent indicator TSA-CY 3.5 and is indicated in red. Images A, B and C represent young, middle-aged and old rat kidneys respectively. Images $\mathrm{G}, \mathrm{H}$, and I represent the outer medulla and medulla of young, middle-aged and old rat kidneys respectively. In these images the red staining has been merged with blue DAPI staining for DNA. Brightfield images were also acquired for the same sections (D-F and J-L). Magnification: 40X; resolution: 300 pixels/inch. Data shown are taken from a single experiment representative of 4 such experiments.

Fig. 4 Microscopy images of kidney cross-sections. PEPT2 staining was measured using the immunofluorescent indicator TSA-CY 3.5 and is indicated in red. Image A cortex and outer medulla of young rat kidneys. Images B and C outer medulla and medulla of middle-aged and old rat kidneys, respectively. Images G, H and I superficial cortex of young, middle-aged and old rat kidneys respectively. DAPI staining DNA (blue) was merged with the specifc-PEPT2 
staining. Brightfield images were also acquired for the same sections (D-F and J-L). Magnification: 40X; resolution: 300 pixels/inch. Data shown are taken from a single experiment representative of 4 such experiments.

Fig 5 Representative Western blotting bands for PEPT1 and PEPT2 proteins with their corresponding $\beta$-actin protein bands. (A) Investigations of the presence/absence of PEPT1 in BBMV isolated from young, middle-aged and old rat kidneys. (B) Investigations of the presence/absence of PEPT2 in OMMV isolated from young, middle-aged and old rat kidneys.

Fig 6 Western blotting bands for PEPT1 and PEPT2 proteins with their corresponding $\beta$-actin protein bands. (A) Investigations of the presence/absence of PEPT1 and PEPT2 in BBMV and OMMV isolated from young rat kidneys. (B) Investigations of the presence of PEPT1 and PEPT2 in BBMV and OMMV isolated from middle-aged and old rat kidneys.

Fig 7 (A) Immunoblot analysis of PEPT1 and (B) PEPT2 proteins in BBMV and OMMV isolated from the kidneys of young, middle-aged and old Wistar rats. The protein concentration loaded in each lane of the gel was constant 45 , or $35 \mu \mathrm{g}$, each of which had its own control ( $\beta$-actin). The relative protein abundance was calculated using autoradiographs, which were subjected to Bio-Rad scanning densitometry. This is followed by calculating the ratio of protein abundance for PEPT to $\beta$-actin (as PEPT1/ $\beta$-actin $\times 100$ ). ${ }^{*} \mathrm{P}<0.01$ vs. all other groups, ${ }^{*} \mathrm{P}<0.05$ vs. middle-aged BBMV or OMMV, and vs. old BBMV and OMMV; and $\# \mathrm{P}<0.05$ vs. old BBMV. Data shown are means $\pm \mathrm{SE}, \mathrm{n}=6-9$

Fig 8 Relative normalised expression of PEPT1 and PEPT2 genes (against two reference genes SDHA, and $\beta$-Actin) measured by QRT-PCR and $\Delta \Delta$ Cq method using Bio-Rad CFX manager 3.1. These genes are relatively quantified in the Cortex (Cox) and Outer medulla (OM) respectively for young adult, middle-aged, and old kidneys of Wistar rat, as colours shown in the graph. ${ }^{*} \mathrm{P}<0.05$ vs. young cortex, ${ }^{*} * \mathrm{P}<0.05$ vs. young and middle-aged outer 
medulla, and $\# \mathrm{P}<0.05$ vs. PEPT1. Data shown are means $\pm \mathrm{SE}, \mathrm{n}=5$ (all samples were run in one 384 well plate and in duplicate)

Table 1: Detailed information of all genes used in this study.

Table 2: Kinetic parameters $\mathrm{Km}(\mu \mathrm{M})$ and $\operatorname{Vmax}(\Delta \mathrm{F} / \mathrm{min} / \mathrm{mg})$ for the uptake of $\beta$-Ala-Lys (AMCA) in BBMV and OMMV isolated from the Kidneys of all Wistar rat group. 